\title{
Implementation of RIPv1 and OSPF Routing Protocol, DHCP, DNS, and HTTP Configurations in CISCO Packet Tracer
}

\author{
Anas Tukur Balarabe ${ }^{1}$, Zahriya Lawal Hassan ${ }^{2}$, Ahmad Rufa' $^{3}{ }^{3}$ \\ Sokoto State University, Sokoto ${ }^{1,2,3}$
}

\begin{abstract}
This paper gives elaborate descriptions on the implementation of two routing protocols namely: Routing Information Protocol (RIP) and Open Shortest Path First (OSPF). It also covers Variable Length Subnet Mask (VLMS) Dynamic Host Configuration Protocol, Hypertext Transfer Protocol, and Domain Name Server configurations. With dynamic routing protocols, a router has the ability to determine which path to select when routing a packet this is achieved through a frequent update of the routing table by the router using information provided by different networks in a giving set up about their locations. The paper also demonstrates how IP addresses are conserved using Variable Length Subnet Masking.
\end{abstract}

Keywords: RIP, OSPF, DHCP, HTTP, DNS

\section{INTRODUCTION}

In any organized and effective system of communication, there must be a mechanism to govern how information is being exchanged between communicating parties. In computer networks, routers route and switch packets across the internet. The routers have a set of rules that they employ in order to perform the routing function, these rules are called protocols in terms of computer networks. Routers use these protocols to make decisions in selecting paths to use in sending a message from a source network to the destination. Jain et al (2014). Computer network connects devices via different communication media such as the fibre, coaxial cable, twisted pair and other forms of unguided media. The end devices connect to the network through the lower layer devices such as switches and hubs. Yousif M.K and Alsaffar S.K (2018). In this paper, Cisco Packet Tracer has been used to implement some selected routing protocols. This paper gives elaborate descriptions on the implementation of two routing protocols namely: Routing Information Protocol (RIP) and Open Shortest Path First (OSPF). It also covers Variable Length Subnet Mask (VLMS) Dynamic Host Configuration Protocol, Hypertext Transfer Protocol, and Domain Name Server configurations. With dynamic routing protocols, a router has the ability to determine which path to select when routing a packet this is achieved through a frequent update of the routing table by the router using information provided by different networks in a giving set up about their locations. The paper also demonstrates how IP addresses are conserved using Variable Length Subnet Masking.

\section{PACKET TRACER}

Dangwal and Kumar (2014) defined Packet Tracer as network simulation software that allows designing, implementation and analysis of different network features for research, learning and some other applications. Cisco System invented this tool for use in teaching and learning of different networking skills. A network is simulated by selecting devices and dropping them in the simulation environment. Devices can be configured through the Command Line Interface (CLI) or Graphical User Interface (GUI). The later provides text fields, checkboxes, and buttons for setting device configuration parameters. Protocols are configured through the CLI by following correct steps and entering correct commands. The version of Packet Tracer used in this research is Cisco Packet Tracer 6.0.1.

\section{DYNAMIC ROUTING}

According to Jain et al (2014), every router has a routing table which holds the network addresses information of other networks. In static routing, routing tables are created manually, however, in dynamic routing as the name implies routing tables are created dynamically, which makes dynamic routing network managers friendly. In this type of routing, routing tables are frequently updated through making each device on the network to broadcast information about its location. This information is then used to maintain the routing tables. Routing algorithms are the forces behind dynamic routing protocols functionalities, these algorithms help routers in decision making on path selection. Some of 


\section{International Journal of Innovative Research in Electrical, Electronics, Instrumentation and Control Engineering}

Vol. 7, Issue 4, April 2019

the dynamic routing algorithms include link state, distance vector, interior, and exterior gateway routing algorithms.

\section{ROUTING INFORMATION PROTOCOL}

In two articles authored by Jain et al (2014) and Thakur (2014), RIP as it is widely known, the Routing Information Protocol is one of the best routing protocols. It belongs to the family of distance vector routing protocols and it is very easy to implement. As one of the early routing protocols, RIP is compatible with all the networking devices. With RIP, routers send routing table information in a space of 30 seconds which is then redistributed from router to router to make all hosts aware of the routing paths on the network. Routing information protocol has two versions namely: RIPv1 and RIPv2. RIPv1 was created in the early 80s and was the only RIP version available until 1993 when v2 was created. RIPv2 was created to address the drawbacks of RIPv1 which does not support Classless Inter-Domain Routing. Because of its small number of hop count, in addition to its 30 seconds interval, constant routing table information dissemination RIP can impose delay and consequently not suitable for use in large networks.

\section{OPEN SHORTEST PATH FIRST}

In their literature review Jain et al (2014), Ramya and Vinod (2012) and Nazamudeen and Mahendra (2014) the authors explained that unlike RIP, OSPF belongs to the Link State protocols family. OSPF allows routers to create maps of connected local networks to determine the shortest path to select when sending packets. Link State protocols used what is called Link State Database to maintain the information of all routers on the network, with this information; the router creates a hierarchical structure of shortest paths to each of the known routers. This is achieved through placing itself at the apex of the hierarchy; the router has a panoramic view of the entire network. One of the measure advantages of Open Shortest Path First (OSPF) is that it supports Variable Subnet Mask (VLMS) and therefore supports Classless Inter-Domain Routing (CIDR). Variable Length Subnet Mask helps Network Administrators conserve IP address by making a reduction of the number of hosts allocated to networks possible.

\section{DYNAMIC HOST CONFIGURATION PROTOCOL}

According to Kurose and Ross (2013), Dynamic Host Configuration Protocol takes away the burden of assigning IP addresses manually to hosts in a given network by allocating IP addresses to the hosts automatically. In addition to temporary IP address allocation, the DHCP permits hosts to acquire addition network details such as default gateway, Domain Name System server address and their subnet mask. Dynamic Host Configuration is more suitable for use in a network where nodes join and leave the network randomly.

\section{DOMAIN NAME SYSTEM PROTOCOL}

Kurose and Rose (2013) explained that FTP and SMT, Domain Name System is an application layer protocol that runs between the communicating devices. DNS, however, performs a different function from other application layer services such as Web, email and File Transfer. Alternatively, the DNS provides an integral internet function, which is translating domain names to their corresponding IP address for user applications and other services on the internet.

\section{HYPERTEXT TRANSFER PROTOCOL (HTTP)}

Kurose and Rose (2013) defined The HTTP as the protocol that governs how programs on the client side and programs on the server site interact. Once a user requests access to a webpage the DNS server receives the domain name and passes its matching IP address to the HTTP server with the request, the HTTP server receives then responds to the request.

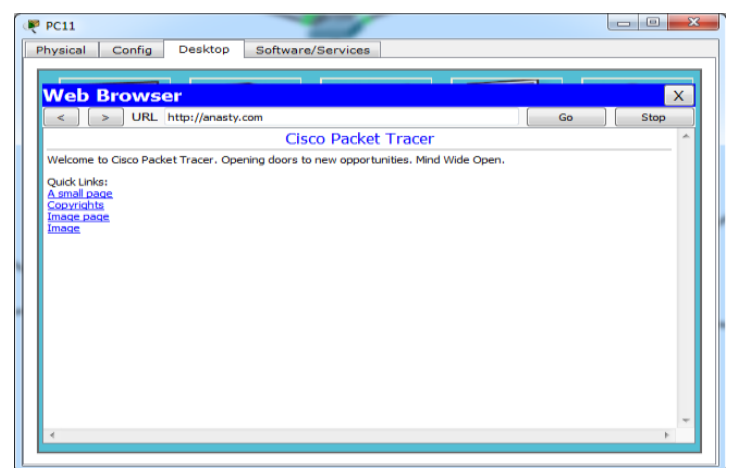

Fig. 1: A Snapshot of PC11 Accessing the HTTP file with Domain Name anasty.com 


\section{IJIREEICE \\ International Journal of Innovative Research in Electrical, Electronics, Instrumentation and Control Engineering}

Vol. 7, Issue 4, April 2019

\section{VARIABLE LENGTH SUBNET MASK (VLSM)}

According to Dangwal and Kumar (2014), through subnetting a given network can be divided into subnetworks, this gives room for greater routing efficiency and reduces unwanted broadcasts that might slow down the network. The Internet Protocol addressing is of two classes namely: Classful Subnetting and Classless Subnetting. Both classes of IP addressing allow subdivision of a network into subnetworks, however Classful subnetting is very rigid because there is no way of reducing the number of hosts per network. For example, with Classful it will not be possible to reduce the number of hosts allocated to a network that will link two routers, even though only four host address can be used. But with classless, it is possible to minimize IP address wastage by reducing the number of host IP allocated to a network. This is achieved through varying the host bits to create subnetworks. Variable Length Subnet Masks allows IP addressing flexibility; it also makes network management easier in addition to network security that can be achieved through segmenting the network.

\section{THE SCENARIO}

To implement these routing protocols a scenario was created and simulated. The scenario is as follows:

A company with four branches was imagined, each branch requires 20 hosts addresses. The Headquarters requires 30 host addresses, and a total of 8 IP addresses are needed for the setup.

- $\quad$ Brach A requires 20 host IP addresses.

- $\quad$ Branch B requires 20 host IP addresses.

- $\quad$ Branch $\mathrm{C}$ requires 20 host IP addresses

- $\quad$ Branch D requires 20 host IP addresses

- $\quad$ Each link between the three routers requires an IP address

- $\quad$ The headquarters requires 30 host IP addresses.

\section{THE SIMULATION PROCESS}

a)

RIPv1 Implementation

Given 192.168.1.0/24 the IP addresses were created for the implementation of RIP which does not support Classless Inter-Domain Routing. The following subnets were created:

$\begin{array}{ll}192.168 .1 .0 / 27 & 192.168 .1 .32 / 27 \\ 192.168 .1 .64 / 27 & 192.168 .1 .96 / 27 \\ 192.168 .1 .128 / 27 & 192.168 .1 .160 / 27 \\ 192.168 .1 .192 / 27 & 192.168 .1 .224 / 27\end{array}$

The subnet mask of the subnetworks is 255.255 .255 .224 .

To set up the topology, the following networking devices were used:

- Generic Router

- Generic Server

- $\quad$ Switch (2950-24)

- $\quad$ Network Printer

- $\quad$ Generic Laptop

- $\quad$ Generic PC

- $\quad$ Serial DCE

- $\quad$ Straight-Through Cable

The routers were connected using serial DCE, straight-through cable is used for connecting PCs to switches, servers to switches, printers to switches, switches to routers and laptops to switches. 
International Journal of Innovative Research in Electrical, Electronics, Instrumentation and Control Engineering

Vol. 7, Issue 4, April 2019

The above devices were connected to generate the topology in fig.1.

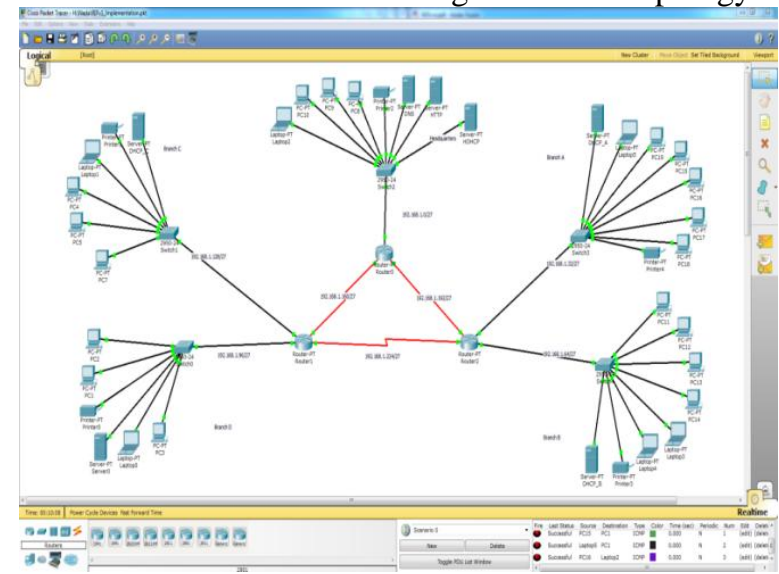

Fig. 2: RIP Configuration with Classful Subnetting.

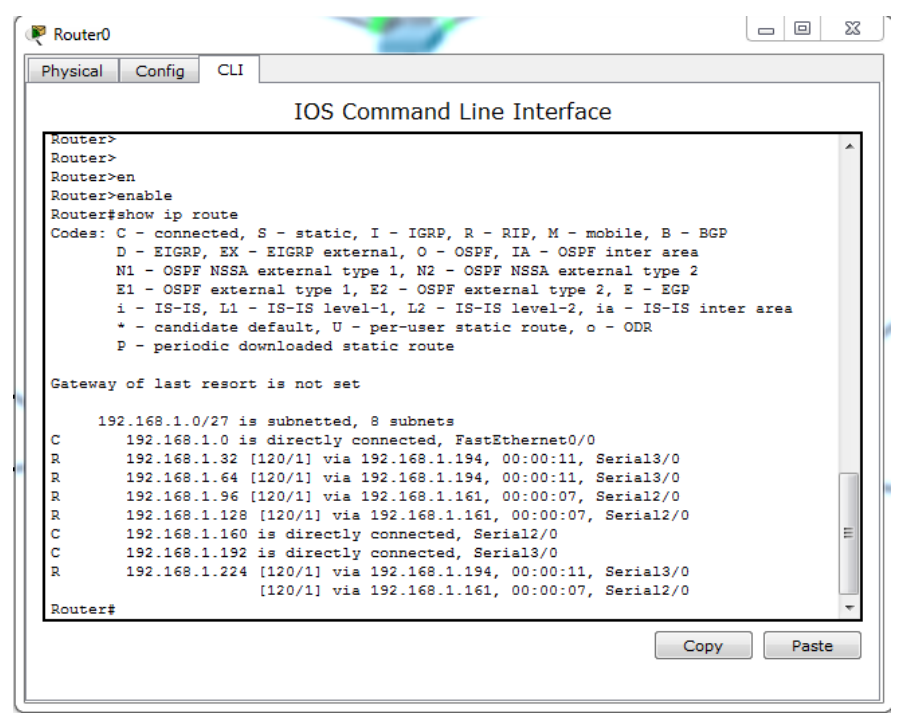

Fig.3: Router0 IP Route Showing Parent Network and Child Networks.

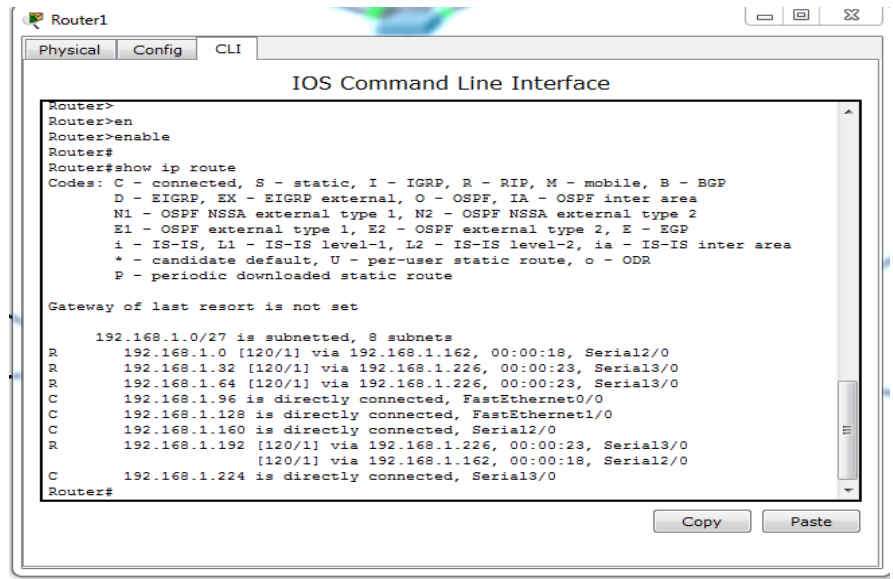

Fig.4: Router 1 Routing Table. 


\section{International Journal of Innovative Research in Electrical, Electronics, Instrumentation and Control Engineering}

Vol. 7, Issue 4, April 2019

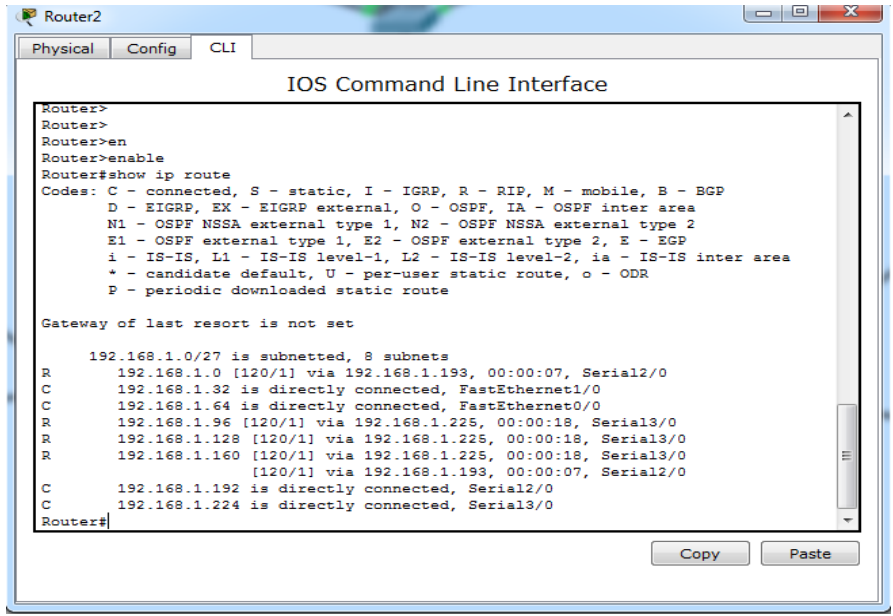

Fig.5: Router 2 Routing Table.

To configure DNS server the following steps were followed:

- Double Click on a connected server to be configured for DNS service

- Click on Config menu

- $\quad$ Click on DNS from the list of services on the left-hand side.

- $\quad$ Add the domain name and the IP address of your site (which is HTTP server IP address) and save.

- Make sure that DNS service is on.

- $\quad$ Click on the desktop menu to enter the DNS server's static IP address and default gateway, by going into the IP configuration icon.

- Close.

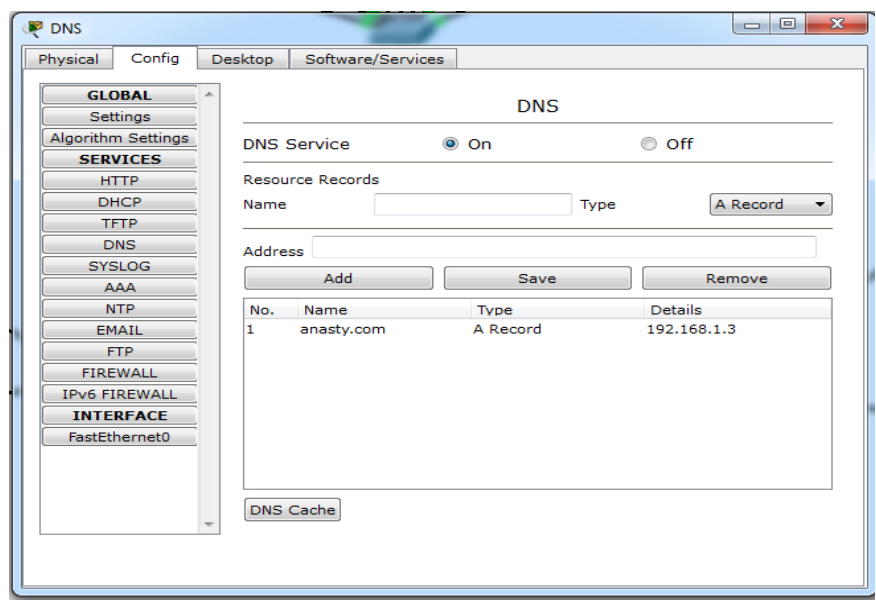

Fig.6: DNS Server Configuration Interface.

To configure HTTP server the following steps were followed:

- Double click on a connected server to be configured for HTTP service

- Click on the Config menu.

- $\quad$ Click on HTTP from the list of services on the left-hand side and make sure that the HTTP service is on.

- Click on the clock on the desktop menu to give the server a static and default gateway IP address by clicking on the configuration icon.

- $\quad$ Close. 


\section{International Journal of Innovative Research in Electrical, Electronics, Instrumentation and Control Engineering}

Vol. 7, Issue 4, April 2019

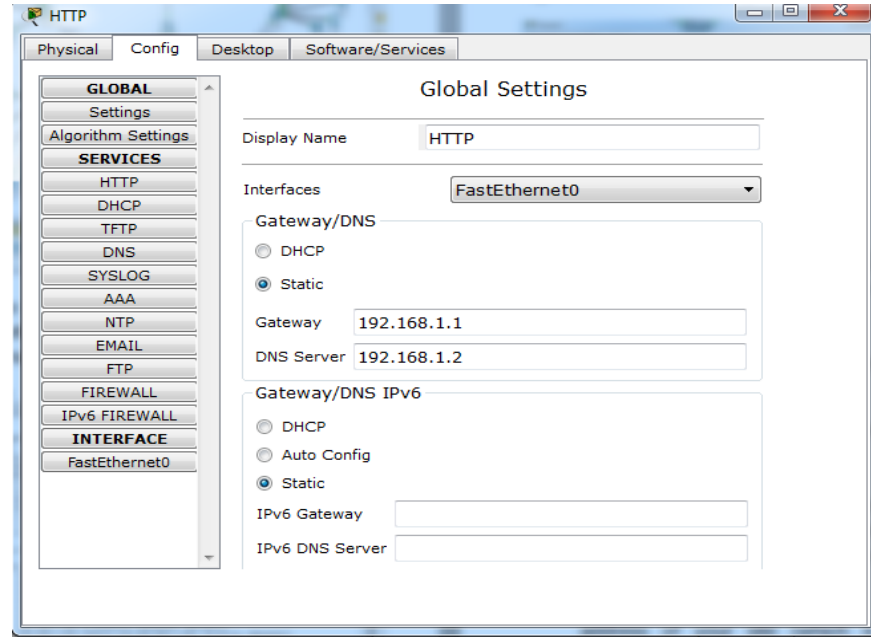

Fig.7: HTTP Server Configuration Interface.

To configure DCH server the following steps were followed

- Double click on a connected server to be configured for DHCP service.

- Click on the Config menu on the menu bar.

- $\quad$ Click on the DHCP from the list of services on the left-hand side.

- $\quad$ Enter the default gateway and DNS server IP addresses.

- $\quad$ Enter the Start IP Address (if the default gateway IP address is 192.168.1.1 and the DCHP server IP address is 192.168.1.2 then the DCHP can be set to start allocating IP addresses from 192.168.1.3 to the last user IP address)

- $\quad$ Enter the Subnet Mask of the start IP address

- $\quad$ Save

- Make sure that the DHCP service is on and then close.

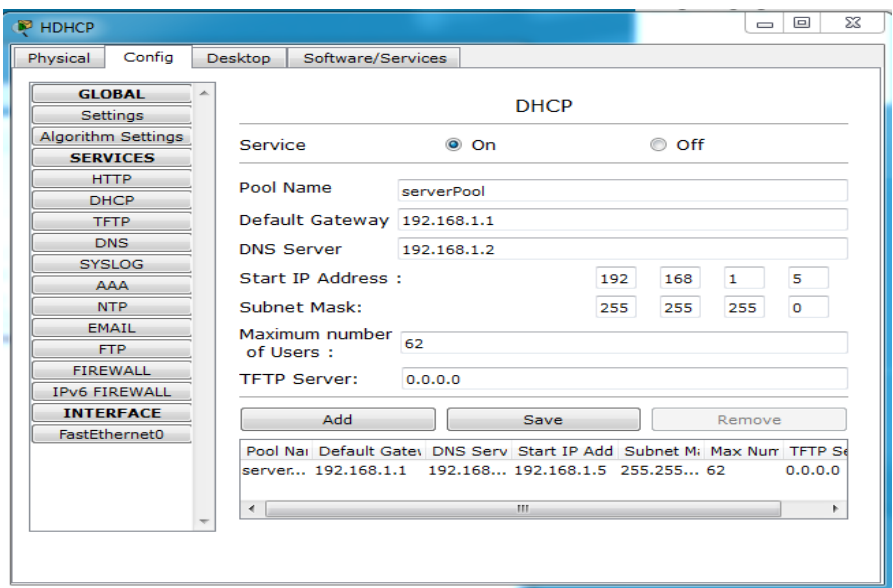

Fig.8: DHCP Server Configuration Interface.

Finally, to make each host acquire IP address from the DHCP server, click on the host (PCs, Laptop or Printer) go to Desktop then click on IP Configuration. In the IP configuration interface activate the radio button with the label DHCP and DHCP Request Successful message will appear with IP addresses of a host, Default Gateway and DNS and Subnet Mask. 
International Journal of Innovative Research in Electrical, Electronics, Instrumentation and Control Engineering

Vol. 7, Issue 4, April 2019

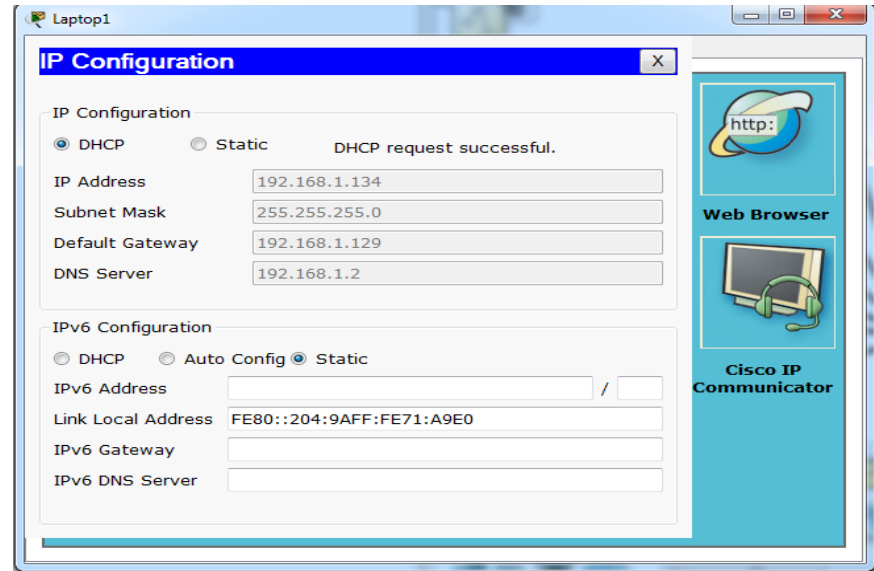

Fig.9: IP Configuration Interface.

\section{b) OSPF Implementation}

With the same scenario used in implementing RIPv1 network address 192.168.1.0/24 was variably subnetted to create the following subnets:

- $\quad$ Headquarters network address

192.168.1.0/27 255.255.255.224

- $\quad$ Four Branches network addresses

192.168.1.32/27 255.255.255.224

192.168.1.64/27 255.255.255.224

192.168.1.96/27 255.255.255.224

192.168.1.128/27 255.255.255.224

Three router interfaces network addresses.

$192.168 .1 .164 / 30 \quad 255.255 .255 .252$

$192.168 .1 .168 / 30 \quad 255.255 .255 .252$

$192.168 .1 .172 / 30 \quad 255.255 .255 .252$

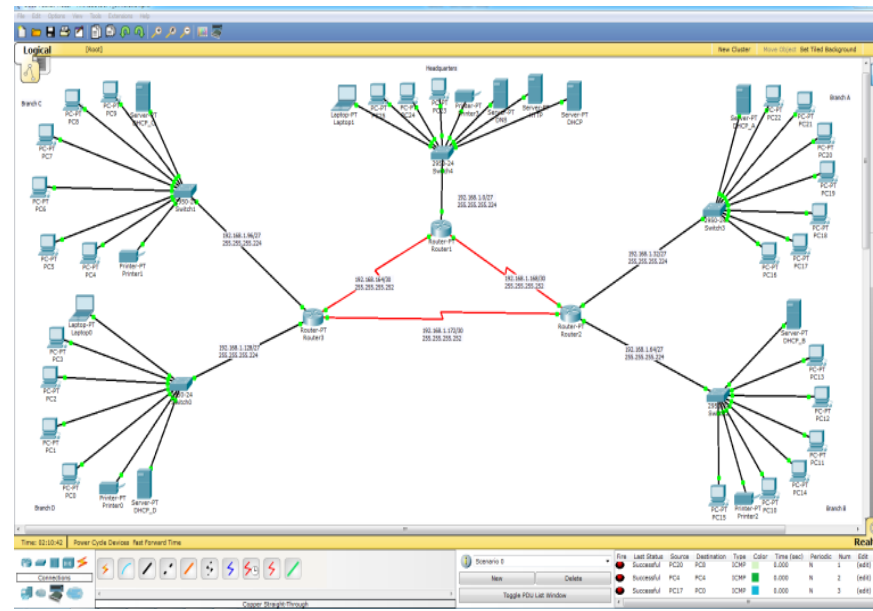

Fig.10: OSPF Configuration using Variable Length Subnet Mask. 


\section{International Journal of Innovative Research in Electrical, Electronics, Instrumentation and Control Engineering}

Vol. 7, Issue 4, April 2019

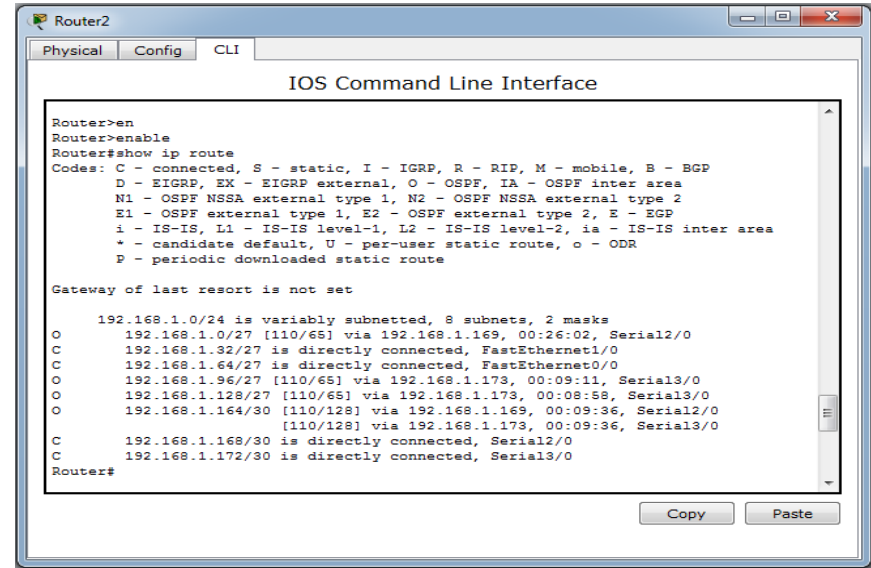

Fig.11: Router2 Parent Network and Child Networks

\section{RESULT \& DISCUSSION}

It can be observed that RIP can be configured using the traditional IP address subnetting scheme. The measure setbacks of this subnetting scheme are: it does not provide flexibility and it also accounts for IP address wastage. The OSPF, on the other hand, supports classless subnetting and CIDR, this feature of OSPF affords the network administrators the ability to utilize and conserve IP addresses by allocating an appropriate number of IP addresses to each subnet.

\section{CONCLUSION}

In this paper, we have used a single scenario to implement two routing protocols, RIPv1 and OSPF. RIPv1 is very simple to implement once there are basic classful subnetting skills and enough knowledge of the participating networks. OSPF implementation is complex, excellent subnetting skills (VLSM) are needed to implement it. Because each IP address has to be entered with its own subnet mask during interfaces configuration. In addition to the use of wildcards when configuring the OSPF, it can be said to be one of the most difficult routing protocols to implement. Finally, the choice of which protocol to implement depends on the nature of the network. If a network is simple and does not involve different classes of IP address and contains not more than 15 hop counts then RIP is the appropriate choice. But for large and complex networks, OSPF should be implemented because of its low bandwidth consumption, ability to support different IP addresses and fast convergence time.

\section{REFERENCES}

[1]. Yousif M.K and Al-saffar S.K (2018) 'Project Scenario of Communication Network using Cisco Packet Tracer'. International Journal of Computer Applications (0975 - 8887), pp.37-47

[2]. Abr td (2012) How to Configure OSPF in Cisco Packet Tracer. Available at: https://www.youtube.com/watch?v=iIRXIEG-6S0 (Accessed: 10 March 2019).

[3]. Andre, N (2012) Lecture-Variable Length Subnet Mask. Available at: https://www.youtube.com/watch?v=IiVpVt5pzsA (Accessed: 10 March 2019).

[4]. Cryo(2012) Packet Tracer Tutorial: Connecting and Configuring Two Routers Using RIP. Available at: https://www.youtube.com/watch?v=JP1zdb234xo (Accessed: 15 March 2019).

[5]. Dangwal, K. and Kumar, V. (2014) 'Comparative Study of EIGRP and RIP Using Cisco Packet Tracer', International Journal of Engineering Sciences \& Emerging Technologies, 6(6), pp.475-480.

[6]. Jain, P. K., Sindhwani, M., and Sachdeva (2014) 'Comparative Study of Routing Protocols with Subnetting Implementation in Cisco Packet Tracer', 4(12), pp. 646-650.

[7]. Kurose, J.F. and Ross, K. W (2013) Computer Networking a Top-Down Approach. $6^{\text {th }}$ edn. Boston: Pearson.

[8]. Nazamudeen, N. and Mahendra, C. (2014) 'Performance Analysis of Dynamic Routing Protocols Using Packet Tracer'. International Journal of Innovative Research in Science, Engineering and Technology, 3(1), pp. 570-574.

[9]. Ramya, P. and Vinod, V (2012) 'Implementing OSPF Protocol in Cisco 2800 Series Router'. International Journal of Innovations in Engineering and Technology, 1(4), pp. 77-82.

[10]. Thakur, P. (2014) 'Evaluation of Dynamic Routing Protocols with Packet Tracer: RIP and EIGRP'. International Journal of Mobile and Adhoc Network (IJMAN), 4(3), pp273-282 